\title{
Work productivity loss in mild to moderate COPD: lessons learned from the CanCOLD study
}

\author{
To the Editor:
}

Little attention has been given to the impact of chronic obstructive pulmonary disease (COPD) on work productivity loss. Individuals with COPD are at risk of reduced working hours, absenteeism, presenteeism and early retirement [1]. Studies have been focused mostly on patients attending outpatient clinics [2], which exclude individuals with undiagnosed COPD, thus limiting the external validity of the findings. There are very few population-based cohort studies [3-6], few reports on presenteeism [5], and a lack of objective measures to define COPD [6]. There would be value in knowing the extent of work productivity loss in individuals with mild COPD, or those who are yet undiagnosed. This could further translate into the allocation of health management programmes in the workplace.

We evaluated work productivity loss (measured as absenteeism and/or presenteeism) between individuals with mild to moderate COPD and those without COPD. We hypothesised that working individuals with COPD, having mild and moderate airflow obstruction with a high symptom burden would have greater work productivity loss than those with a low symptom burden.

Our study was embedded in the Canadian Cohort Obstructive Pulmonary Disease (CanCOLD) study, an ongoing multicentre study involving subjects with COPD, sampled from the general population (see BOURBEAu et al. [7], for full details). For the purpose of the current study, inclusion criteria for employed COPD and non-COPD control subjects were as follows: $\geqslant 40$ years old, having paid employment at the time of study enrolment and a post-bronchodilator forced expiratory volume in $1 \mathrm{~s} /$ forced vital capacity $(\mathrm{FEV} 1 / \mathrm{FVC})<0.70$ for COPD versus $\mathrm{FEV} 1 / \mathrm{FVC} \geqslant 0.70$ for non-COPD. All participants provided written informed consent and the study was approved by the respective institutional ethical review boards. Subjects with spirometrically defined COPD, who reported prior physician-diagnosed COPD, chronic bronchitis, or emphysema upon entering the CanCOLD study were identified as "diagnosed" subjects, and subjects with spirometrically defined COPD, who had not received a COPD diagnosis prior to enrolment in the study, were identified as having "undiagnosed" COPD. The overall work productivity loss, i.e. absenteeism and/or presenteeism, was measured by the health and labour questionnaire (HLQ) [8], a generic and validated instrument. Absenteeism refers to health-related absence from work, whereas presenteeism refers to the act of attending work while sick, e.g. decreased work quality or quantity. Symptom burden was measured using the COPD Assessment Test (CAT) [9]. Subjects with CAT scores $<10$ were classified as low symptom burden and those with CAT scores $\geqslant 10$ as high symptom burden [10]. Comparisons between groups for descriptive summaries were performed using ANOVA or the Kruskal-Wallis test for continuous variables, and the Chi-squared test for dichotomous variables. The level of significance was set at $\mathrm{p}<0.05$. All analyses were carried out using the SAS version 9.1.3 software (SAS Institute Inc., Cary, NC, USA).

Of 1556 subjects recruited from the CanCOLD study, 1484 completed spirometry and the HLQ. The study population consisted of 207 employed COPD subjects and 271 non-COPD control subjects. Overall, subjects were on average 60 years old; $60 \%$ were female and more than $50 \%$ had $>2$ comorbidities. The COPD subjects, in comparison to the non-COPD controls, had a longer smoking history $(21.8 \pm 25.1$ versus 11.7 \pm 16.6 pack-years; $\mathrm{p}<0.001$ ), greater airflow obstruction ( $\mathrm{FEV} 1 / \mathrm{FVC}$ of $62.2 \pm 6.9$ versus $77.6 \pm 5.1 \%$; $\mathrm{p}<0.001$ and $\mathrm{FEV}_{1} \%$ predicted of $82.1 \pm 17.0$ versus $\left.100.1 \pm 15.9 ; \mathrm{p}<0.001\right)$, and both showed relatively good

@ERSpublications

COPD patients with high symptom burden or CAT $\geqslant 10$ have an increased likelihood of experiencing work productivity loss http://ow.ly/FnG030e6uaY

Cite this article as: de Sousa Sena R, Ahmed S, Tan WC, et al. Work productivity loss in mild to moderate COPD: lessons learned from the CanCOLD study. Eur Respir J 2017; 50: 1701154 [https://doi. org/10.1183/13993003.01154-2017]. 




FIGURE 1 Proportion of work productivity loss in the past month for chronic obstructive pulmonary disease (COPD) and non-COPD subjects categorised according to high symptom burden (COPD Assessment Test (CAT) score $\geqslant 10$ ) and low symptom burden (CAT score $<10$ ).

health status with CAT scores of $6.5 \pm 5.5$ versus $5.4 \pm 4.6$. Only $23 \%$ of the COPD subjects had a physician diagnosis of COPD.

Figure 1 illustrates the work productivity loss (absenteeism, presenteeism and the two combined) in COPD and non-COPD subjects, according to high $(\mathrm{CAT} \geqslant 10)$ and low symptom burdens $(\mathrm{CAT}<10)$. Work productivity loss in subjects with a high symptom burden was about 3-fold higher than in COPD subjects with a low symptom burden and in non-COPD subjects. Among the COPD subjects reporting presenteeism, the most frequent type of impairment reported was difficulty in concentrating (64\%) and a slower than usual pace at work (64\%), followed by difficulties making decisions (57\%), postponement of work (36\%) and the need for assistance (21\%). Absenteeism was higher in subjects with physician-diagnosed COPD compared to those with undiagnosed COPD ( $14.6 \%$ versus $5.7 \%, p=0.04)$. However, the difference was not significant for presenteeism $(4.2 \%$ versus $7.5 \%, \mathrm{p}=0.41)$ and overall work productivity loss $(18.9 \%$ versus $11.9 \%, \mathrm{p}=0.22)$.

The present study provides a comprehensive overview of work productivity loss (absenteeism and/or presenteeism) among a representative population-based sample of individuals with mild to moderate COPD. Despite having relatively mild to moderate airflow obstruction, and on average a health status comparable to healthy control subjects, we demonstrated that COPD subjects with a high symptom burden $(\mathrm{CAT} \geqslant 10$ ), experience increased work productivity loss (i.e. combined absenteeism and presenteeism) compared to COPD subjects with a low symptom burden (CAT <10), and non-COPD subjects.

In keeping with our results, Foo et al. [11] reported that COPD subjects who experienced a greater degree of breathlessness and other symptoms showed high work productivity loss. Consistent with our findings, JANSson et al. [5] showed that absence from work owing to respiratory diseases was higher in subjects with diagnosed COPD compared to those with undiagnosed COPD. This could be explained by the fact that a physician diagnosis of COPD usually identifies those experiencing a greater number of respiratory symptoms and/or exacerbations that could result in increased absence from work [5]. Thus, strategies to reduce the societal burden of COPD should include those that prevent both hospitalisations and work-related outcomes by optimising medication use, self-management interventions and pulmonary rehabilitation.

One of the strengths of the present study is the selection of subjects with COPD from a random population sample, thus making it more representative of the COPD population at large. This provides information not only on individuals with physician diagnosed COPD, but those that are still undiagnosed, who represent $70 \%$ of the individuals with COPD. In addition, COPD diagnosis was based on an objective 
assessment of post-bronchodilator spirometry, in contrast with previous studies that relied on self-reporting or screening of symptoms $[12,13]$. Another strength of the present study is the inclusion of a comparison group of subjects with non-COPD. This allowed enhanced estimation of the magnitude of difference in work productivity loss in COPD subjects. Furthermore, both presenteeism and absenteeism were reported in the current study on COPD with mild to moderate airflow obstruction, thereby overcoming the limitations of previous studies on COPD that focused on absenteeism alone $[4,12]$.

There are also limitations that should be considered in the interpretation of our results. First, the sample size of employed subjects was relatively small and might have led to an underestimation of the true effects of COPD. Recall and social desirability biases cannot be excluded, considering the work productivity evaluation was done using questionnaires.

In conclusion, our study shows that the burden of COPD goes beyond its impact on the health care system. There is potential for a significant burden at the workplace, even among individuals with mild to moderate airflow obstruction. This study also emphasises the importance of assessing symptom burden, to identify those with a high symptom burden, or CAT $\geqslant 10$, who have an increased likelihood of experiencing increased work productivity loss (i.e., combined absenteeism and presenteeism). There is a need for further studies to evaluate the relevance of early COPD recognition by physicians, particularly in those patients who are still in the workforce, and consider whether optimal pharmacological and non-pharmacological management would reduce symptoms, improve work performance, and reduce societal cost.

Riany de Sousa Sena ${ }^{1,2}$, Sara Ahmed ${ }^{1}$, Wan C. Tan ${ }^{3}$, Pei Z. Li $^{1}$, Laura Labonté ${ }^{1}$, Shawn D. Aaron ${ }^{4}$, Andrea Benedetti ${ }^{1}$, Kenneth R. Chapman ${ }^{5}$, B. Walker ${ }^{6}$, J. Mark Fitzgerald ${ }^{3}$, Paul Hernandez ${ }^{7}$, François Maltais ${ }^{8}$, Darcy D. Marciniuk ${ }^{9}$, Denis E. O'Donnell ${ }^{10}$, Don D. Sin $^{3}$, Jean Bourbeau ${ }^{1}$ and the CanCOLD Collaborative Research Group and The Canadian Respiratory Research Network ${ }^{11}$

${ }^{1}$ Respiratory Epidemiology and Clinical Research Unit, Research Institute of the McGill University Health Centre, McGill University, Montreal, QC, Canada. ${ }^{2}$ University of Fortaleza, Fortaleza, Brazil. ${ }^{3}$ University of British Columbia, Vancouver, BC, Canada. ${ }^{4}$ Ottawa University, Ottawa, ON, Canada. ${ }^{5}$ University of Toronto, Toronto, ON, Canada. ${ }^{6}$ University of Calgary, Calgary, AB, Canada. ${ }^{7}$ Dalhousie University, Halifax, NS, Canada. ${ }^{8}$ Institut universitaire de cardiologie et de pneumologie de Québec, Université Laval, Québec, QC, Canada. ${ }^{9}$ University of Saskatchewan, Saskatoon, SK, Canada. ${ }^{10}$ Queens University, Kingston, ON, Canada. ${ }^{11} \mathrm{~A}$ list of members of the CanCOLD Collaborative Research Group can be found in the Acknowledgements section.

Correspondence: Jean Bourbeau, Respiratory Epidemiology and Clinical Research Unit, 5252 De Maisonneuve West, Montréal, Quebec, H4A 3S5, Canada. E-mail: jean.bourbeau@mcgill.ca

Received: April 142017 | Accepted after revision: June 162017

Support statement: The Canadian Cohort Obstructive Lung Disease (CanCOLD) study is currently funded by the Canadian Respiratory Research Network (CRRN); industry partners: Astra Zeneca Canada Ltd; Boehringer Ingelheim Canada Ltd; GlaxoSmithKline Canada Ltd; and Novartis. Researchers at RI-MUHC Montreal and Icapture Centre Vancouver lead the project. Previous funding partners are the CIHR (CIHR/Rx\&D Collaborative Research Program Operating Grants 93326); the Respiratory Health Network of the Fonds de la recherche en santé du Québec (FRSQ); industry partners: Almirall; Merck Nycomed; Pfizer Canada Ltd; and Theratechnologies. The funders had no role in study design, data collection and analysis, or preparation of the manuscript. Funding information for this article has been deposited with the Crossref Funder Registry.

Conflict of interest: Disclosures can be found alongside this article at erj.ersjournals.com

Acknowledgements: The authors thank the men and women who participated in the study and individuals in the CanCOLD Collaborative Research Group.

Author contributions: R. de Sousa Sena contributed to the conception and implementation of the study, analysis of the data, and writing of the manuscript. J. Bourbeau contributed to the study conception and design, implementation and acquisition of the data, and writing and revision of the article. S. Ahmed contributed to the study conception and design and the writing and revision of the article. W.C. Tan, L. Labonté, S.D. Aaron, A. Benedetti, K.R. Chapman, B. Walker, J.M. Fitzgerald, P. Hernandez, F. Maltais, D.D. Marciniuk, D.E. O'Donnell and D.D. Sin contributed to the acquisition of data and revision of the article. P.Z. Li contributed to the analysis and interpretation of the data. All authors approved the final version of the manuscript.

Members of the CanCOLD Collaborative Research Group are as follows. Executive Committee: Jean Bourbeau (McGill University, Montreal, Canada); Wan C. Tan, J. Mark FitzGerald; Don Sin (UBC, Vancouver, Canada); Darcy Marciniuk (University of Saskatoon, Saskatoon, Canada); Dennis E. O'Donnell (Queen's University, Kingston, Canada); Paul Hernandez (Dalhousie University, Halifax, Canada); Kenneth R. Chapman (University of Toronto, Toronto, Canada); Robert Cowie (University of Calgary, Calgary, Canada); Shawn Aaron (University of Ottawa, Ottawa, Canada); F. Maltais (University of Laval, Quebec City, Canada). International Advisory Board: Jonathon Samet (Keck School of Medicine of USC, Los Angeles, CA); Milo Puhan (John Hopkins School of Public Health, Baltimore, MD); Qutayba Hamid (McGill University, Montreal, Canada); James C. Hogg (UBC James Hogg Research Center, Vancouver, Canada). Operations Center: Jean Bourbeau (PI), Carole Jabet, Palmina Mancino, (McGill University, Montreal, Canada); Wan C. Tan (co-PI), Don Sin, Sheena Tam, Jeremy Road, Joe Comeau, Adrian Png, Harvey Coxson, Jonathon Leipsic, Cameron Hague (University of British Columbia James Hogg Research Center, Vancouver, Canada). Economic 
Core: Mohsen Sadatsafavi (University of British Columbia, Vancouver, Canada). Public Health Core: Teresa To, Andrea Gershon (University of Toronto, Toronto, Canada). Data Management and Quality Control: Wan C. Tan, Harvey Coxson (UBC, Vancouver, Canada); Jean Bourbeau, Pei Zhi Li, Zhi Song, Yvan Fortier, Andrea Benedetti, Dennis Jensen (McGill University, Montreal, Canada). Field Centers: Wan C. Tan (Vancouver PI), Christine Lo, Sarah Cheng, Cindy Fung, Nancy Haynes, Junior Chuang, Licong Li, Selva Bayat, Amanda Wong, Zoe Alavi, Catherine Peng, Bin Zhao, Nathalie Scott-Hsiung, Tasha Nadirshaw (UBC James Hogg Research Center, Vancouver, Canada); Jean Bourbeau (Montreal PI), Palmina Mancino, David Latreille, Jacinthe Baril, Laura Labonté (McGill University, Montreal, Canada); Kenneth Chapman (Toronto PI), Patricia McClean, Nadeen Audisho (University of Toronto, Toronto, Canada); R. Cowie and B. Walter (Calgary PI), Ann Cowie, Curtis Dumonceaux, Lisette Machado (University of Calgary, Calgary, Canada); Paul Hernandez (Halifax PI), Scott Fulton, Kristen Osterling (Dalhousie University, Halifax, Canada); Shawn

Aaron (Ottawa PI), Kathy Vandemheen, Gay Pratt, Amanda Bergeron (University of Ottawa, Ottawa, Canada); Denis O’Donnell (Kingston PI), Matthew McNeil, Kate Whelan (Queen's University, Kingston, Canada); François Maltais (Quebec PI), Cynthia Brouillard (Université Laval, Quebec City, Canada); Darcy Marciniuk (Saskatoon PI), Ron Clemens, Janet Baran (University of Saskatoon, Saskatoon, Canada).

\section{References}

1 Chaker L, Falla A, van der Lee SJ, et al. The global impact of non-communicable diseases on macro-economic productivity: a systematic review. Eur J Epidemiol 2015; 30: 357-395.

2 Andenaes R, Bentsen SB, Hvinden K, et al. The relationships of self-efficacy, physical activity, and paid work to health-related quality of life among patients with chronic obstructive pulmonary disease (COPD). J Multidiscip Healthc 2014; 7: 239-247.

3 Montes de Oca M, Halbert RJ, Talamo C, et al. Paid employment in subjects with and without chronic obstructive pulmonary disease in five Latin American cities: the PLATINO study. Int J Tuberc Lung Dis 2011; 15: 1259-1264, i-iii.

4 Erdal M, Johannessen A, Askildsen JE, et al. Productivity losses in chronic obstructive pulmonary disease: a population-based survey. BMJ Open Respir Res 2014; 1: e000049.

5 Jansson SA, Backman H, Stenling A, et al. Health economic costs of COPD in Sweden by disease severity--has it changed during a ten years period? Respir Med 2013; 107: 1931-1938.

6 Landis SH, Muellerova H, Mannino DM, et al. Continuing to Confront COPD International Patient Survey: methods, COPD prevalence, and disease burden in 2012-2013. Int J Chron Obstruct Pulmon Dis 2014; 9: 597-611.

7 Bourbeau J, Tan WC, Benedetti A, et al. Canadian Cohort Obstructive Lung Disease (CanCOLD): fulfilling the need for longitudinal observational studies in COPD. COPD 2014; 11: 125-132.

8 Van Roijen L, Essink-Bot ML, Koopmanschap MA, et al. Labor and health status in economic evaluation of health care. The Health and Labor Questionnaire. Int J Technol Assess Health Care 1996; 12: 405-415.

9 Gupta N, Pinto LM, Morogan A, et al. The COPD assessment test: a systematic review. Eur Respir J 2014; 44: 873-884.

10 Jones PW, Tabberer M, Chen WH. Creating scenarios of the impact of COPD and their relationship to COPD Assessment Test (CAT) scores. BMC Pulm Med 2011; 11: 42.

11 Foo J, Landis SH, Maskell J, et al. Continuing to Confront COPD International Patient Survey: economic impact of COPD in 12 countries. PloS One 2016; 11: e0152618.

12 Darkow T, Chastek BJ, Shah H, et al. Health care costs among individuals with chronic obstructive pulmonary disease within several large, multi-state employers. J Occup Environ Med 2008; 50: 1130-1138.

13 DiBonaventura M, Paulose-Ram R, Su J, et al. The burden of chronic obstructive pulmonary disease among employed adults. Int J Chron Obstruct Pulmon Dis 2012; 7: 211-219. 